\title{
Hypoxia Induced High Expression of Thioredoxin Interacting Protein (TXNIP) in Non-small Cell Lung Cancer and its Prognostic Effect
}

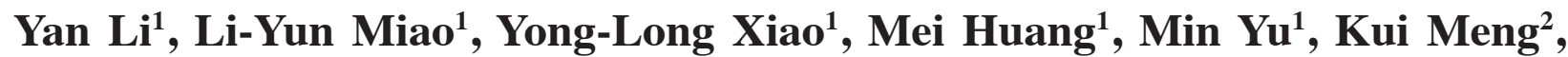 \\ Hou-Rong Cai ${ }^{1 *}$
}

\begin{abstract}
Although associations between thioredoxin interacting protein (TXNIP) and cancers have been recognized, the effects of TXNIP on non-small cell lung cancer (NSCLC) prognosis remained to be determined in detail. In addition, while hypoxia is a key characteristic of tumor cell growth microenvironment, the effect of hypoxia on TXNIP expression is controversial. In this study, formaldehyde fixed and paraffin embedded (FFPE) samples of 70 NSCLC patients who underwent resection between January 2010 and December 2011 were obtained. Evaluation of TXNIP and hypoxia inducible factor-1 $\alpha(\mathrm{HIF}-1 \alpha)$ protein expression in FFPE samples was made by immunohistochemistry. By Kaplan-Meier method, patients with high TXNIP expression demonstrated a significantly shorter progression free survival (PFS) compared with those with low TXNIP expression (18.0 months, 95\% CI: 11.7, 24.3 versus 23.0 months, $95 \%$ CI: $17.6,28.4, P=0.02$ ). High TXNIP expression level was also identified as an independent prognostic factor by Cox regression analysis (adjusted hazard ratio: 2.46; 95\% CI: 1.08, 5.56; $P=0.03$ ). Furthermore, TXNIP expression was found to be significantly correlated with HIF$1 \alpha$ expression (Spearman correlation $=0.67, P=0.000$ ). To further confirm correlations, we established a tumor cell hypoxic culture model. Expression of TXNIP was up-regulated in all three NSCLC cell lines (A549, SPC-A1, and H1299) under hypoxic conditions. This study suggests that hypoxia induces increased TXNIP expression in NSCLC and high TXNIP expression could be a poor prognostic marker.
\end{abstract}

Keywords: Thioredoxin interacting protein - hypoxia inducible factor-1 $\alpha$ - survival - prognosis

Asian Pac J Cancer Prev, 16 (7), 2953-2958

\section{Introduction}

Thioredoxin interacting protein (TXNIP), also known as vitamin D3 upregulated protein (VDUP-1) or thioredoxin binding protein-2 (TBP-2), was originally reported as a gene of unknown function in HL-60 cells induced by $1 \alpha, 25$-dihydroxyvitamin D3 (Chen and DeLuca, 1994). The human TXNIP gene is located on chromosome 1q21.1, contains 8 exons and is 4174 bp in length. The human TXNIP protein consists of 391 amino acids and has a molecular mass of $46 \mathrm{kDa}$. TXNIP belongs to alpha-arrestin protein family and is perhaps the only family member known to bind thioredoxin (TRX). TXNIP plays an important role in a wide variety of biological functions, such as the regulation of cell death, growth, differentiation, and energy metabolism (Aitken et al., 2004; Lee et al., 2005; Ahsan et al., 2006; Corbett, 2008; Oka et al., 2009). TXNIP is an important gene that is known to be transcriptionally regulated in response to hypoxia (Wong and Hagen, 2013). However, the effect of hypoxia on TXNIP expression in Non-small cell lung cancer (NSCLC) remained undefined.

One of the tumor progression characteristics is the tumor cell growth microenvironment turns to be a hypoxia condition. Tumor growth, invasion, energy metabolism and incompletion of the local blood vessels lead to a hypoxic state in local tissue. Tissue hypoxia induces a sustained increase in the expression of hypoxia inducible factor-1 (HIF-1). HIF-1 plays an important role in maintaining the energy metabolism of tumor cells, blood vessel expansion, angiogenesis, and cell proliferation (Fraga et al., 2009). The HIF-1 $\beta$ subunit is constitutively expressed whereas the HIF-1 $\alpha$ subunit is oxygen regulated (Semenza, 2011). Regulation of HIF-1 is determined by the rapid posttranslational degradation or stabilization of the HIF- $1 \alpha$ subunit. It has been widely reported that HIF-1 $\alpha$ was highly expressed in NSCLC and related with poorer prognosis of NSCLC patients (Ping et al., 2013; Wang et al., 2014). However, the prognostic effect of TXNIP in NSCLC needs to be further determined. 
The aim of our study was to investigate the expression and the prognostic effect of TXNIP in NSCLC patients. Furthermore, the relationship between TXNIP and HIF$1 \alpha$ was studied. In addition, the influence of hypoxia microenvironment on TXNIP expression in NSCLC cell lines was also explored.

\section{Materials and Methods}

\section{Patients and tissue samples}

Formaldehyde fixed and paraffin embedded (FFPE) NSCLC samples of 70 patients who underwent surgery in the department of thoracic surgery, the Affiliated Drum Tower Hospital of Nanjing University Medical School (Nanjing, China) between January 2010 and December 2011 were retrospectively studied. All the pathology diagnoses were reviewed by two senior pathologists (K Meng and J Yang) according to the WHO criteria (Brambilla et al., 2001). After pathological diagnoses, pTNM stages of 70 patients were designated according to the 7th edition of the TNM classification of malignant tumors (Goldstraw et al., 2007). The detailed data of all the patients showed in Table 1 . The study was approved by the Ethic Committee of Drum Tower Hospital and each patient gave written informed consent.

\section{Immunohistochemistry (IHC)}

Evaluation of TXNIP and HIF-1 $\alpha$ protein expression in FFPE samples was detected by IHC. Consecutive $3 \mu \mathrm{m}$ sections were cut and placed on polylysine coated slides for further analysis. Each paraffin section was deparaffinized and rehydrated through dimethylbenzene and graded alcohols. After the slides were prepared, antigen retrieval was carried by pressure cooking the slides with citric acid buffer ( $\mathrm{pH} 6.0$ for $1 \mathrm{~min}$ ). $3 \%$ $\mathrm{H} 2 \mathrm{O} 2$ was used to block the endogenous peroxidase activity. To block nonspecific staining, the slides were incubating with $5 \%$ bovine serum albumin (BSA) in phosphate buffer saline (PBS) for $15 \mathrm{~min}$. The adjacent two slides were then incubated overnight at $4{ }^{\circ} \mathrm{C}$ with mouse monoclonal TXNIP antibody (1:100 dilution, MBL, Japan), and rabbit polyclonal HIF1- $\alpha$ antibody (1:100 dilution, Abcam, USA), respectively. In order to reduce variability, all samples from each group were stained at the same time in a single experiment using a single batch of antibody diluted in PBS with BSA. Slides were then washed by PBS and incubated with biotinylated goat anti-rabbit IgG antibody (1:500 dilution, Abcam, USA) for half an hour at $37^{\circ} \mathrm{C}$. The reaction product was developed by diaminobenzidine tetrahydrochloride. At the end, the slides were counterstained with hematoxylin. Thereafter, the tissues were washed in distilled water for 5 minutes, dehydrated sequentially and mounted in resinous mountant. Positive controls were included in each run, as well as negative controls were performed with no primary antibody.

\section{Evaluation of TXNIP/HIF-1 $\alpha$ expression}

Evaluation of TXNIP and HIF-1 $\alpha$ expression was independently performed by two independent pathologists who were blinded to clinical data. The immunostaining intensity and the percentage of positive cells distribution of TXNIP and HIF-1 $\alpha$ were calculated for further analysis. Percentages of positive tumor cells defined in at least five areas by $400 \times$ magnification were averaged. The mean percentage was then designed to five gradations: $0,<5 \%$; $1,5 \%-25 \% ; 2,26 \%-50 \% ; 3,51 \%-75 \%$; and $4,>75 \%$. Staining intensity was calculated and marked as follows: weak, 1; moderate, 2; and intense, 3 . The intensity and percentage scores were multiplied to yield a composite score of 0 to 12 for each sample. Composite scores of 0 to 3 were defined as indicating low TXNIP or low HIF-1 $\alpha$ expression respectively, while scores of 4 to 12 were considered to indicate high TXNIP or high HIF-1 $\alpha$ expression.

\section{Cell culture and hypoxia model}

NSCLC cell lines (A549, SPC-A1 and H1299), human umbilical vascular endothelial cell line (HUVEC) and human fetal lung fibroblast cell line (HFL-1) were purchased from ATCC and maintained in our laboratory. All the cell lines were cultured in recommended growth medium in $37^{\circ} \mathrm{C}$ and $5 \% \mathrm{CO}_{2}$. As previously reported (Shi et al., 2013), a hypoxic cell culture model was established with hypoxic chamber in our laboratory. Three NSCLC cell lines, A549, SPC-A1 and H1299 were cultured under two different oxygen concentrations, $1 \%$ and $20 \%$, respectively. For three-dimensional (3D) cell culture, each NSCLC cell line $\left(1 \times 10^{6}\right)$ was mixed with HUVEC cells $\left(1 \times 10^{6}\right)$ and HFL- 1 cells $\left(1 \times 10^{6}\right)$ in Matrigel (BD Bioscience, USA). After 24 hours culture, cells were collected and total RNA were extracted by Trizol (Invitrogen, USA) for reverse transcription-polymerase chain reaction (RT-PCR) analyses.

\section{Real time RT-PCR}

cDNA was synthesized from $2 \mu \mathrm{g}$ of total RNA and random hexamers using the Takara Reverse Transcription kit (Takara, Japan). Quantitative RT-PCR was performed in 48-well plates using a StepOne Real-time PCR system (ABI, USA). All reactions were performed in triplicate and verified by melting curve analysis. The relative amount of mRNA in each sample was normalized to GAPDH transcript levels. Primer sequences were as follows: TXNIP, forward: 5'-AGAGCCAACAGAACAGAAGAA-3' and reverse: 5'-AGAGGCAGATCATTTAAGAGTG-3'; GAPDH, forward: 5' - CAATGACCCCTTCATTGACC -3' and reverse: 5' - TGGAAGATGGTGATGGGATT -3'.

\section{Follow-up visit of patients}

Patients with NSCLC were interviewed by telephone. The progression (recurrence or metastasis) of NSCLC was confirmed by radiology examination or medical record collected from the Drum Tower Hospital. Progression free survival (PFS) time was defined as the time elapsed since the resection of the tumor to the date of disease progression or death.

\section{Statistical analysis}

Differences between clinicopathological variables and the expression of TXNIP were examined by Chi-square test (or Fisher's exact test if any sample number was less 
Hypoxia Induced High Expression of Thioredoxin Interacting Protein (TXNIP) in NSCLC and its Prognostic Effect

than 5). Correlation between TXNIP and HIF-1 $\alpha$ was carried out by Spearman Correlation. Survival curves were calculated using the Kaplan-Meier method and compared by the log-rank test. Multivariate Cox regression model analysis was established to assess the prognostic values of protein expression. Relative expression of TXNIP in cultured NSCLC cells was expressed as mean \pm SEM. Statistical significance was determined by one-way ANOVA, LSD and unpaired t tests. All analyses were performed with SPSS software, version 16.0 (SPSS, Inc., Chicago, IL, USA). All tests were two-sided and performed at a significance level of 0.05 .

\section{Results}

Expression of TXNIP was related with HIF-1 $\alpha$ expression in NSCLC tumor samples

HIF- $1 \alpha$ was up-regulated in $51.4 \%(36 / 70)$ NSCLC tumor samples in our study and HIF- $1 \alpha$ was mainly located in tumor cell nucleus as indicated in Figure 1b. While TXNIP protein was located mainly in cytoplasm of NSCLC cells as shown in Figure 1d. A total of 45 patients $(64.3 \%)$ showed TXNIP high expression. Interestingly, the distribution of HIF- $1 \alpha$ expression in tumor section was significantly related with the distribution of TXNIP expression (Figure 1a and Figure 1c both negative; Figure $1 \mathrm{~b}$ and Figure 1d both positive in the same tumor section). In addition, expression level of TXNIP was significantly correlated with HIF-1 $\alpha$ expression level (Figure 1e, Spearman Correlation $=0.67, P=0.000$ ).

TXNIP expression was up-regulated in NSCLC cell lines under hypoxic condition

To further confirm the relationship between the expression of TXNIP and that of HIF- $1 \alpha$ in NSCLC, we established a tumor cell hypoxic culture model. Expression of TXNIP was up-regulated in all three NSCLC cell lines (A549, SPC-A1, and H1299) under hypoxic culture condition (1\% O2) (Figure 2a). To mimic the tumor microenvironment, we mixed each of three NSCLC cells

with HUVEC and HFL-1 cells in Matrigel (Figure 2b). Consistent with common culture, expression of TXNIP was up-regulated in all three NSCLC 3D culture models under hypoxic culture condition $(1 \% \mathrm{O} 2)$ (Figure $2 \mathrm{c})$.

\section{Patients with high TXNIP expression level had poorer prognosis}

The median PFS of 70 included NSCLC patients was 19.0 months (95\%CI: 16.9, 21.1). Clinicopathological characteristics of NSCLC patients are listed in Table 1 by TXNIP expression level. There were no significant differences in age, gender, cell type, and pTNM stage between patients with high and low TXNIP expression (Ps $>0.05)$. The median PFS in patients with high HIF-1 $\alpha$ expression was significantly shorter than that in those with low HIF-1 $\alpha$ expression [14.0 months (95\%CI: 10.0, 18.0) vs 23.0 months (95\%CI: $20.5,25.5), P=0.002]$. Similarly, the median PFS was 18.0 months $(95 \% \mathrm{CI}$ : 11.7, 24.3) in patients with high TXNIP expression and 23.0 months

Table 1. Characteristics of 70 Non-small Cell Lung Cancer Patients

\begin{tabular}{lccc}
\hline Patient Characteristics & \multicolumn{2}{c}{ TXNIP Expression } & P value \\
& $\begin{array}{c}\text { High (45) } \\
\text { No. }(\%)\end{array}$ & $\begin{array}{c}\text { Low (25) } \\
\text { No. (\%) }\end{array}$ & \\
& & & \\
\hline Age & $18(40)$ & $10(40)$ & 1.00 \\
$\quad<60$ years old & $27(60)$ & $15(60)$ & \\
$\quad \geq 60$ years old & & & 0.40 \\
Gender & $10(22.2)$ & $8(32.0)$ & \\
$\quad$ Female & $35(77.8)$ & $17(68.0)$ & \\
$\quad$ Male & & & 0.44 \\
Cell type & $30(66.7)$ & $14(56.0)$ & \\
$\quad$ Adenocarcinoma & $15(33.3)$ & $11(44.0)$ & \\
$\quad$ Squamous & & & 0.98 \\
pTNM stage & $22(48.9)$ & $12(48.0)$ & \\
I & $13(28.9)$ & $7(28.0)$ & \\
II & $10(22.2)$ & $6(24.0)$ & \\
III & & & $<0.001$ \\
HIF-1 $\alpha$ expression & $34(75.6)$ & $2(8.0)$ & \\
$\quad$ High & $11(24.4)$ & $23(92.0)$ & \\
Low & & & \\
\hline
\end{tabular}
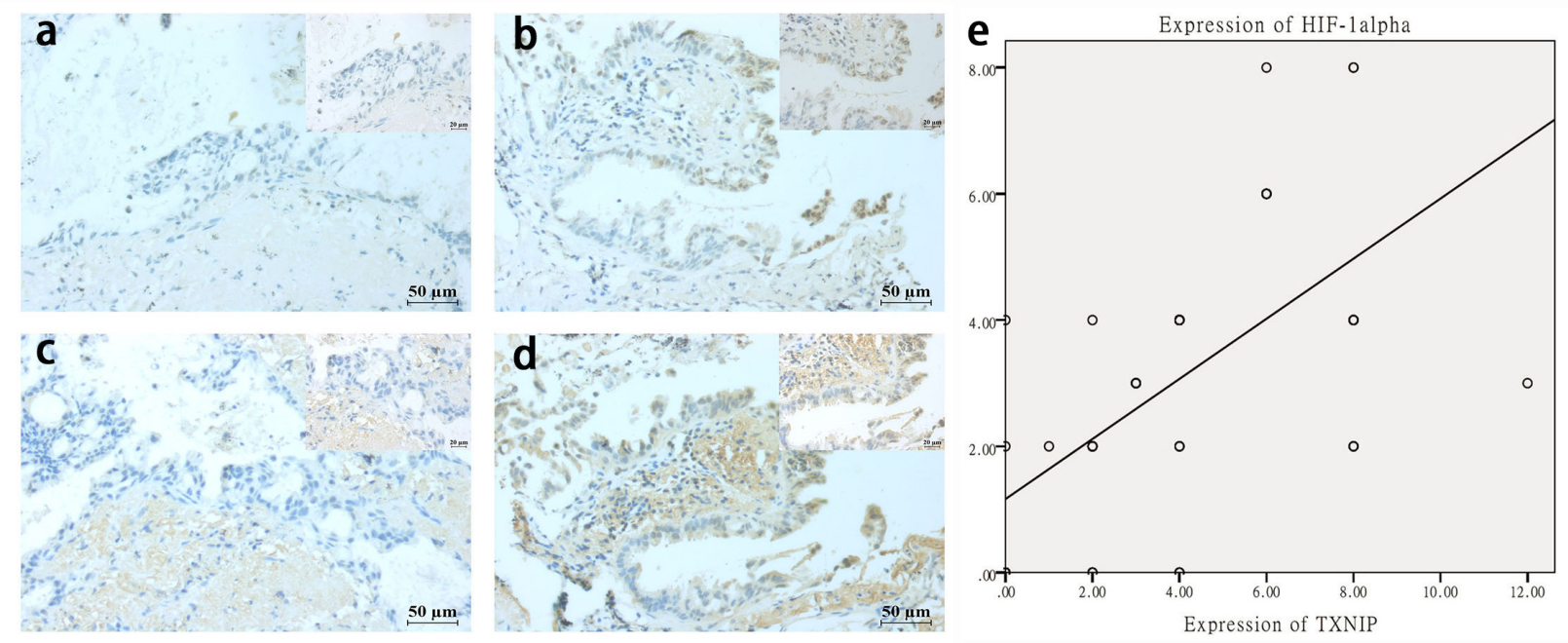

Figure 1. Expression of TXNIP and HIF-1 $\alpha$ in NSCLC Tumor Samples. a and b, Negative and positive expression of HIF- $1 \alpha$ on the same section of one sample. $c$ and d, Negative and positive expression of TXNIP at the same positions on the same sections as in a and b correspondingly. e, The relation between TXNIP expression and HIF-1 $\alpha$ expression, Spearman Correlation $=0.673$, $P=0.000$. Scale bar, as indicated in the figure 

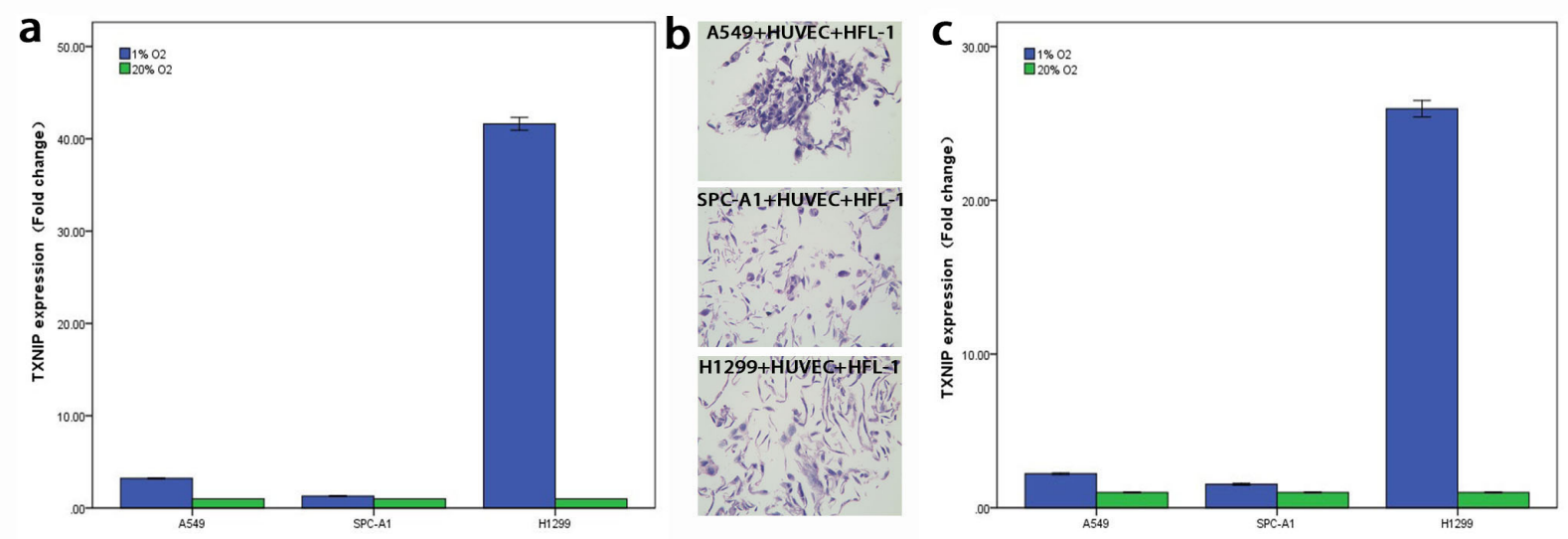

Figure 2. Expression of TXNIP in NSCLC Cell Lines Under Hypoxic Culture Condition. NSCLC cells alone or mixed with interstitial cells were cultured under different oxygenic conditions $\left(1 \% \mathrm{O}_{2}\right.$ and $\left.20 \% \mathrm{O}_{2}\right)$. a, Expression of TXNIP was up-regulated in all three NSCLC cell lines (A549, SPC-A1, and H1299) under hypoxic culture condition (1\% $\left.\mathrm{O}_{2}\right)$. b, H\&E staining of the mixed culture (3D culture model) of NSCLC cells with interstitial cells (human umbilical vascular endothelial cell (HUVEC) and human fetal lung fibroblast cell (HFL-1)). It could mimic the real tumor microenvironment. c, Expression of TXNIP was upregulated in all three NSCLC 3D culture models under hypoxic culture condition $\left(1 \% \mathrm{O}_{2}\right)$
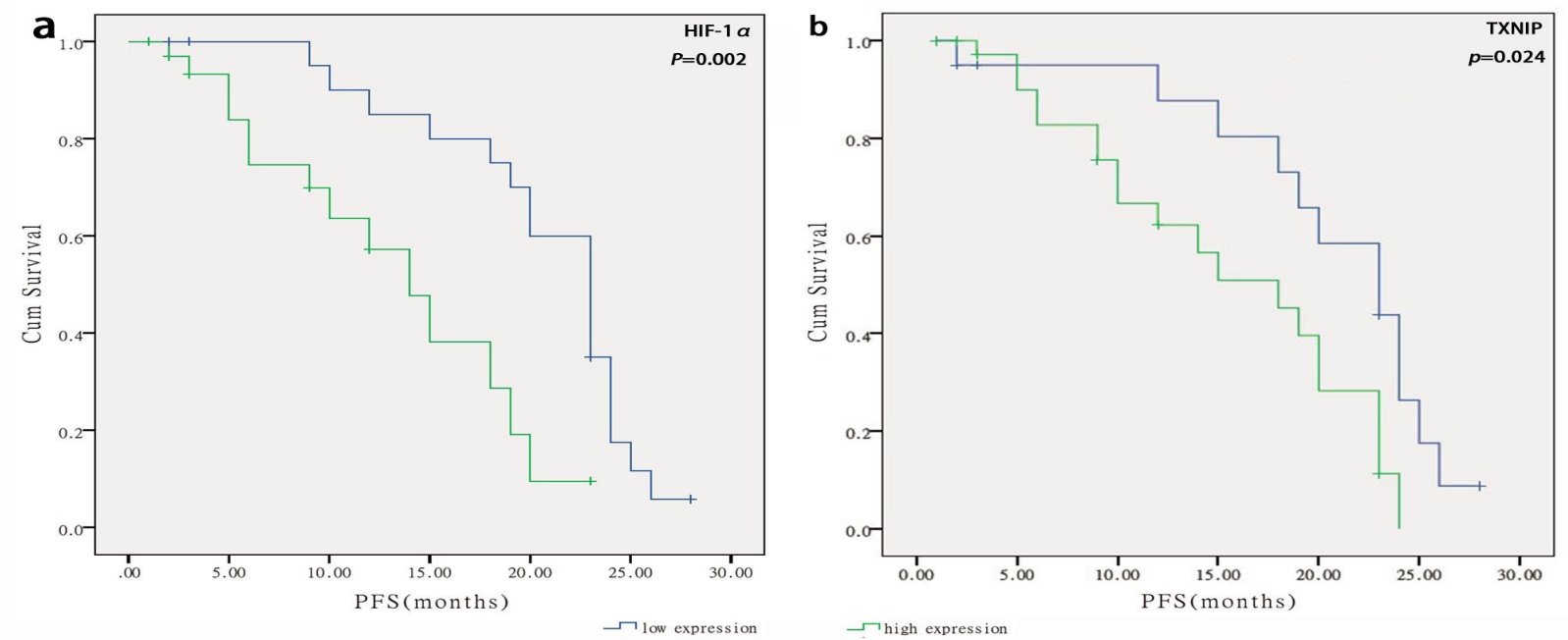

Figure 3. Prognostic Effects of TXNIP and HIF-1 $\alpha$ in NSCLC. a, The median PFS in patients with high HIF-1 $\alpha$ expression was significantly shorter than that in those with low HIF-1 $\alpha$ expression [14.0 months (95\% CI: 10.0, 18.0) vs 23.0 months (95\% CI: 20.5, 25.5), $\mathrm{P}=0.002] . \mathrm{b}$, The median PFS was 18.0 months (95\% CI: 11.7, 24.3) in patients with high TXNIP expression and 23.0 months $(95 \%$ CI: 17.6, 28.4) in those with low TXNIP expression $(\mathrm{P}=0.02)$

(95\%CI: 17.6, 28.4) in those with low TXNIP expression. The Kaplan-Meier PFS curve showed a significant separation $(P=0.02$, Figure 3$)$. In univariate analysis, there was a significant association of TXNIP expression level with PFS (hazard ratio: 2.32; 95\%CI: $1.05,5.16 ; P=0.04$ ). In multivariate analysis adjusting for age, gender, cell type, and pTNM stage, the significance remained (hazard ratio: 2.46; 95\%CI: $1.08,5.56 ; P=0.03)$.

\section{Discussion}

In present study, we showed high TXNIP expression was associated with a shorter PFS in NSCLC patients. More importantly, our study demonstrated that TXNIP expression was related with HIF-1 $\alpha$ expression in NSCLC and up-regulated under hypoxic condition.

Hypoxia inducible factor-1 (HIF-1) is the first identified mediator of cell response to hypoxia in mammalian cells cultured under reduced oxygen tension (Semenza and Wang, 1992). This transcription factor is a heterodimer composed of two subunits: an oxygen-sensitive HIF-1 $\alpha$ and a constitutively expressed HIF- $1 \beta$. The subunits of HIF-1 bind together to acquire transcriptional properties, allowing it to regulate the transcriptional activity of hundreds of genes that promote cancer cell survival in hypoxic conditions. Levels of HIF- $1 \alpha$ protein increase exponentially as oxygen concentration declines (Semenza, 2011). One of the tumor progression characteristics is the tumor cell growth microenvironment turns to be a hypoxia condition. Tumor growth, invasion, energy metabolism and incompletion of the local blood vessels lead to a hypoxic state in local tissue. TXNIP has been shown to be a hypoxia-induced gene in human microvascular endothelial cells (Le Jan et al., 2006) and in murine heart (Karar et al., 2007). TXNIP has also been reported to be induced during hypoxia in a HIF-dependent manner in pancreatic cancer (Baker et al., 2008). In our study, TXNIP expression was up-regulated in NSCLC cell lines under hypoxic condition, which is consistent with previous studies. The mechanism through which TXNIP expression 
was regulated by hypoxia has not been confirmed. Some report showed the regulation of TXNIP in hypoxia was mediated via the inhibition of 4E-BP1/eIF4E axis of mTORC1 (Wong and Hagen, 2013). On the other hand, TXNIP may also have some effect on HIF-1 $\alpha$ expression. Some study reported that TXNIP might mediate HIF-1 $\alpha$ translocation from the nucleus to the cytoplasm and target HIF-1 $\alpha$ to degradation (Shin et al., 2008). These results suggested that there might be more complex mechanisms involving TXNIP and HIF-1 $\alpha$ in cancer cell survival, which need further investigating.

TXNIP was originally discovered by virtue of its strong regulation by vitamin D. Expression of TXNIP was upregulated in HL-60 cells stimulated with $1 \alpha$, 25-dihydroxyvitamin D3, and was augmented by glucose, adenosine-containing molecules, the HDAC inhibitor SAHA, 5-fluorouracil, deprivation of serum or IL-2 in cell culture, carcinogens, ceramide, etoposide, hydrogen peroxide $\left(\mathrm{H}_{2} \mathrm{O}_{2}\right)$, heat shock, TGF- $\beta$, ultraviolet light, glucocorticoid, allose treatment and LPS (Masutani et al., 2012). Expression level of TXNIP is influenced by a variety of conditions, suggesting different roles in different biological processes including apoptosis, growth, and differentiation. Some reports have shown downregulation of TXNIP expression in tumor cells and could be upregulated by some anticancer agent (Yoshioka et al., 2006). However, high expression of TXNIP was here observed in NSCLC cell lines and was associated with shorter PFS in NSCLC patients.

Many apoptotic stimuli including anisomysin, oxidative stress, and suberoylanilide hydroxamic acid could induce the rapid upregulation of TXNIP (Butler et al., 2002). However, the effects of enforced TXNIP overexpression on apoptosis were different depending on cell types. In NIH3T3 fibroblasts, SNU stomach tumor cells, and 293 cells, TXNIP overexpression was not sufficient to induce apoptosis (Junn et al., 2000). Previous studies that used a high concentration of reactive oxygen species (ROS) indicated TXNIP was proapoptotic via activation of multiple death pathways (Chen et al., 2008). However, recent data show that in response to physiological levels of ROS, TXNIP is actually prosurvival (World et al., 2011). TXNIP promotes inflammation in endothelial cells (EC) by binding to thioredoxin-1 (TRX1) in a redox-dependent manner. The TXNIP-TRX1 complex acts as a redox-sensitive mediator to control VEGFR2 signaling, thereby promoting cell survival under conditions of oxidative stress. Different associations of TXNIP in distinct tumor cells imply that TXNIP might play multiple roles.

In conclusion, we investigated the expression of TXNIP in NSCLC and correlated the expression level of TXNIP with poor prognosis. Although it is obviously necessary to study much more cases, these findings suggest that TXNIP might be a useful biomarker to predict the prognosis of NSCLC patients. We also showed that TXNIP was induced in response to hypoxia in NSCLC. A better understanding of the regulatory mechanisms controlling TXNIP expression may prove beneficial in the development of additional therapeutic strategies to modulate TXNIP in cancer.

\section{Acknowledgements}

The work was supported by grant from Natural Science Foundation of Jiangsu Province (BK20130089 to Yan Li). The authors would like to thank Jun Yang, MD., (Department of Pathology, The Affiliated Drum Tower Hospital of Nanjing University Medical School, Nanjing, China) for his technical assistance in the evaluation of the immunostained samples.

\section{References}

Ahsan MK, Masutani H, Yamaguchi Y, et al (2006). Loss of interleukin-2-dependency in HTLV-I-infected T cells on gene silencing of thioredoxin-binding protein-2. Oncogene, 25, 2181-91.

Aitken CJ, Hodge JM, Nishinaka Y, et al (2004). Regulation of human osteoclast differentiation by thioredoxin binding protein-2 and redox-sensitive signaling. J Bone Miner.Res, 19, 2057-64.

Baker AF, Koh MY, Williams RR, et al (2008). Identification of thioredoxin-interacting protein 1 as a hypoxia-inducible factor 1alpha-induced gene in pancreatic cancer. Pancreas, 36, $178-86$.

Brambilla E, Travis WD, Colby TV, Corrin B, Shimosato Y (2001). The new World Health Organization classification of lung tumours. Eur Respir J, 18, 1059-68.

Butler LM, Zhou X, Xu WS, et al (2002) The histone deacetylase inhibitor SAHA arrests cancer cell growth, up-regulates thioredoxin-binding protein-2, and down-regulates thioredoxin. Proc Natl Acad Sci USA, 99, 11700-5.

Chen J, Hui ST, Couto FM, et al (2008). Thioredoxin-interacting protein deficiency induces $\mathrm{Akt} / \mathrm{Bcl}-\mathrm{xL}$ signaling and pancreatic $\beta$-cell mass and protects against diabetes. FASEB $J, 22,3581-94$.

Chen KS, DeLuca HF (1994). Isolation and characterization of a novel cDNA from HL-60 cells treated with 1, 25-dihydroxyvitamin D-3. Biochim Biophys Acta, 1219, 26-32.

Corbett JA (2008). Thioredoxin-interacting protein is killing my beta-cells!. Diabetes, 57, 797-8.

Fraga A, Ribeiro R, Medeiros R (2009). Tumor hypoxia: the role of HIF. Actas Urol Esp, 33, 941-51.

Goldstraw P, Crowley J, Chansky K, et al (2007). The IASLC Lung Cancer Staging Project: proposals for the revision of the TNM stage groupings in the forthcoming (seventh) edition of the TNM classification of malignant tumours. $J$ Thorac Oncol, 2, 706-14.

Junn E, Han SH, Im JY, et al (2000). Vitamin D3 up-regulated protein 1 mediates oxidative stress via suppressing the thioredoxin function. J Immunol, 164, 6287-95.

Karar J, Dolt KS, Mishra MK, et al (2007). Expression and functional activity of pro-oxidants and antioxidants in murine heart exposed to acute hypobaric hypoxia. FEBS Lett, 581, 4577-82.

Lee KN, Kang HS, Jeon JH, et al (2005). VDUP1 is required for the development of natural killer cells. Immunity, 22, 195-208.

Le Jan S, Le Meur N, Cazes A, et al (2006). Characterization of the expression of the hypoxia-induced genes neuritin, TXNIP and IGFBP3 in cancer. FEBS Lett, 580, 3395-400.

Masutani H, Yoshihara E, Masaki S, Chen Z, Yodoi J (2012). Thioredoxin binding protein (TBP)-2/Txnip and $\alpha$-arrestin proteins in cancer and diabetes mellitus. J Clin Biochem Nutr, 50, 23-34.

Oka S, Yoshihara E, Bizen-Abe A, et al (2009). Thioredoxin 
binding protein-2/thioredoxin-interacting protein is a critical regulator of insulin secretion and peroxisome proliferatoractivated receptor function. Endocrinology, 150, 1225-34.

Ping W, Jiang WY, Chen WS, Sun W, Fu XN (2013). Expression and significance of hypoxia inducible factor- $1 \alpha$ and lysyl oxidase in non-small cell lung cancer. Asian Pac J Cancer Prev, 14, 3613-8.

Semenza GL (2011). Hypoxia. Cross talk between oxygen sensing and the cell cycle machinery. Am J Physiol Cell Physiol, 301, 550-2.

Semenza GL, Wang GL (1992). A nuclear factor induced by hypoxia via de novo protein synthesis binds to the human erythropoietin gene enhancer at a site required for transcriptional activation. Mol Cell Biol, 12, 5447-54.

Semenza GL (2011). Regulation of metabolism by hypoxiainducible factor 1. Cold Spring Harb Symp Quant Biol, 76, 347 .

Shi S, Wang R, Chen Y, et al (2013). Combining antiangiogenic therapy with adoptive cell immunotherapy exerts better antitumor effects in non-small cell lung cancer models. PLoS One, 8, 65757.

Shin D, Jeon JH, Jeong M, et al (2008). VDUP1 mediates nuclear export of HIF1alpha via CRM1-dependent pathway. Biochim Biophys Acta, 1783, 838-48.

Wang Q, Hu DF, Rui Y, et al (2014). Prognosis value of HIF$1 \alpha$ expression in patients with non-small cell lung cancer. Gene, 541, 69-74.

Wong RW, Hagen T (2013). Mechanistic target of rapamycin (mTOR) dependent regulation of thioredoxin interacting protein (TXNIP) transcription in hypoxia. Biochem Biophys Res Commun, 433, 40-6.

World C, Spindel ON, Berk BC (2011). Thioredoxin-interacting protein mediates TRX1 translocation to the plasma membrane in response to tumor necrosis factor- $\alpha$ : a key mechanism for vascular endothelial growth factor receptor-2 transactivation by reactive oxygen species. Arterioscler Thromb Vasc Biol, 31, 1890-7.

Yoshioka J, Schreiter ER, Lee RT (2006). Role of thioredoxin in cell growth through interactions with signaling molecules. Antioxid Redox Signal, 8, 2143-51. 